POS PROCEEDINGS

\title{
An Overview of the MHONGOOSE Survey: Observing Nearby Galaxies with MeerKAT
}

W.J.G. de Blok ${ }^{* 1,2,3}$

E-mail: blokeastron.nl

E.A.K. Adams ${ }^{1,3}$, P. Amram ${ }^{4}$, E. Athanassoula ${ }^{4}$, I. Bagetakos ${ }^{1,3}$, C. Balkowski ${ }^{5}$, M.A. Bershady $^{6}$, R. Beswick ${ }^{7}$, F. Bigiel ${ }^{8}$, S.-L. Blyth ${ }^{2}$, A. Bosma ${ }^{4}$, R.S. Booth ${ }^{9}$, A.

Bouchard $^{10}$, E. Brinks ${ }^{11}$, C. Carignan ${ }^{2}$, L. Chemin ${ }^{12}$, F. Combes ${ }^{5}$, J. Conway ${ }^{13}$, E.C. Elson $^{14}$, J. English ${ }^{15}$, B. Epinat ${ }^{4}$, B.S. Frank ${ }^{2}$, J. Fiege ${ }^{15}$, F. Fraternali ${ }^{16,3}$, J.S. Gallagher $^{6}$, B.K. Gibson ${ }^{17}$, G. Heald ${ }^{18}$, P.A. Henning ${ }^{19}$, B.W. Holwerda ${ }^{20}$, T.H. Jarrett $^{2}$, H. Jerjen ${ }^{21}$, G.I. Józsa ${ }^{22,23,24}$, M. Kapala ${ }^{2}$, H.-R. Klöckner ${ }^{25}$, B.S.

Koribalski $^{18}$, R.C. Kraan-Korteweg ${ }^{2}$, S. Leon ${ }^{26}$, A. Leroy ${ }^{27}$, S.I. Loubser ${ }^{28}$, D.M. Lucero $^{3}$, S.S. McGaugh ${ }^{29}$, G.R. Meurer ${ }^{30}$, M. Meyer ${ }^{30}$, M. Mogotsi ${ }^{31}$, B. Namumba ${ }^{2}$, S-H. Oh ${ }^{32}$, T.A. Oosterloo ${ }^{1,3}$, D.J. Pisano ${ }^{33,34}$, A. Popping ${ }^{30}$, S. Ratcliffe ${ }^{22}$, J.A. Sellwood $^{35}$, E. Schinnerer ${ }^{36}$, A.C. Schröder ${ }^{31}$, K. Sheth ${ }^{37}$, M.W.L. Smith ${ }^{38}$, A. Sorgho ${ }^{2}$, K. Spekkens ${ }^{39}$, S. Stanimirovic ${ }^{6}$, K. van der Heyden ${ }^{2}$, W. van Driel $^{5}$, L. Verdes-Montenegro ${ }^{40}$, F. Walter ${ }^{36}$, T. Westmeier ${ }^{30}$, E. Wilcots ${ }^{6}$, T. Williams ${ }^{31}$, O.I. Wong $^{30}$, P.A. Woudt ${ }^{2}$, A. Zijlstra ${ }^{41}$ 
${ }^{1}$ ASTRON Netherlands Institute for Radio Astronomy, the Netherlands

${ }^{2}$ Univ. of Cape Town, South Africa

${ }^{3}$ Kapteyn Institute, Univ. of Groningen, the Netherlands

${ }^{4}$ Lab. Astroph. Marseille France

5 Obs. de Paris, France

${ }^{6}$ Univ. of Wisconsin, USA

7 Jodrell Bank, UK

${ }^{8}$ Univ. Heidelberg, Germany

${ }^{9}$ HartRAO, South Africa

${ }^{10}$ McGill Univ., Canada

${ }^{11}$ Univ. of Hertfordshire, UK

${ }^{12}$ Univ. de Antofagasta, Chile

${ }^{13}$ Chalmers Univ., Sweden

${ }^{14}$ Univ. of Western Cape, South Africa

${ }^{15}$ Univ. Manitoba, Canada

16 Univ. Bologna, Italy

${ }^{17}$ E.A. Milne Center for Astrophysics, Univ. of Hull, UK

18 CSIRO Astronomy and Space Science, ATNF, Australia

${ }^{19}$ Univ. New Mexico, USA

${ }^{20}$ Univ. Louisville, USA

${ }^{21}$ RSAA, ANU, Australia

${ }^{2}$ SKA-SA, South Africa

${ }^{23}$ Rhodes Univ., South Africa

${ }^{24}$ Argeländer-Institut für Astronomy, Germany

${ }^{25}$ MPIfR, Bonn, Germany

${ }^{26}$ ESO, Chile

27 Ohio State Univ., USA

${ }^{28}$ North-West Univ., South Africa

${ }^{29}$ Case Western Reserve Univ., USA

${ }^{30}$ ICRAR/UWA, Australia

${ }^{31}$ SAAO, South Africa

${ }^{32}$ KASI, South Korea

${ }^{33}$ West Virginia Univ., USA

${ }^{34}$ Center for Gravitational Wave and Cosmology, Morgantown, WV, USA

35 Steward Observatory, USA

${ }^{36}$ MPIA, Heidelberg, Germany

${ }^{37}$ NASA, Washington DC, USA

${ }^{38}$ Cardiff Univ., UK

${ }^{39}$ RMC, Canada

${ }^{40}$ IAA, Spain

${ }^{41}$ Univ. Manchester, UK 
MHONGOOSE is a deep survey of the neutral hydrogen distribution in a representative sample of 30 nearby disk and dwarf galaxies with $\mathrm{H}$ I masses from $\sim 10^{6}$ to $\sim 10^{11} M_{\odot}$, and luminosities from $M_{R} \sim 12$ to $M_{R} \sim-22$. The sample is selected to uniformly cover the available range in $\log \left(M_{H I}\right)$. Our extremely deep observations, down to H I column density limits of well below $10^{18} \mathrm{~cm}^{-2}$ - or a few hundred times fainter than the typical $\mathrm{H}$ I disks in galaxies — will directly detect the effects of cold accretion from the intergalactic medium and the links with the cosmic web. These observations will be the first ever to probe the very low-column density neutral gas in galaxies at these high resolutions.

Combination with data at other wavelengths, most of it already available, will enable accurate modeling of the properties and evolution of the mass components in these galaxies and link these with the effects of environment, dark matter distribution, and other fundamental properties such as halo mass and angular momentum.

MHONGOOSE can already start addressing some of the SKA-1 science goals and will provide a comprehensive inventory of the processes driving the transformation and evolution of galaxies in the nearby universe at high resolution and over 5 orders of magnitude in column density. It will be a Nearby Galaxies Legacy Survey that will be unsurpassed until the advent of the SKA, and can serve as a highly visible, lasting statement of MeerKAT's capabilities.

MeerKAT Science: On the Pathway to the SKA

25-27 May, 2016

Stellenbosch, South Africa

${ }^{*}$ Speaker. 


\section{Introduction}

One of the Key Science Questions for the Square Kilometre Array (SKA) is "How do galaxies assemble and evolve?" The SKA will be able to trace the gradual transformation from primordial neutral hydrogen (H I) into galaxies over cosmic time. However, direct, detailed and resolved observations of the sub-kpc-scale physical processes that cause this transformation, taking place both inside and around these evolving galaxies, will probably stay beyond our reach — even with the SKA - for a large span of cosmic time due to resolution and sensitivity limitations.

The only place where a comprehensive survey of the "Galactic ecosystem" can be made is the nearby universe; only locally can we study, in detail, the "baryon cycle", i.e., the flow of gas into galaxies, its physical conditions, its transformation into stars, and how it, in turn, is affected by feedback. Resolved H I observations will tell us how galaxies acquire their gas, how star formation is sustained and, ultimately, how the dark and visible matter together determine and regulate the evolution of galaxies.

Local galaxies are the "fossil records" of the distant, high-redshift galaxies, and provide a wealth of information that will help refine models of galaxy formation and evolution. They provide the foundations on which studies of higher redshift galaxies must be built.

In 2010, time was allocated on MeerKAT to carry out MHONGOOSE ${ }^{1}$, a deep H I survey of 30 nearby galaxies. The MHONGOOSE observations aim to reach a $3 \sigma$ column density limit of $7.5 \cdot 10^{18} \mathrm{~cm}^{-2}$, at a resolution of $30^{\prime \prime}$ and integrated over $16 \mathrm{~km} \mathrm{~s}^{-1}$ (roughly the width of the $\mathrm{H} \mathrm{I}$ line). At $90^{\prime \prime}$ resolution the corresponding $3 \sigma$ limit is $5.5 \cdot 10^{17} \mathrm{~cm}^{-2}$.

The large number of short baselines of MeerKAT will efficiently detect low column density material. Compared to telescopes like the VLA or WSRT, MeerKAT can in a single pointing map the H I twice as far out into a galaxy's halo, providing information on evolutionary processes away from the star forming disks.

MeerKAT will also be the most efficient telescope for producing detailed maps of the highresolution $\left(\sim 6^{\prime \prime}\right) \mathrm{H}$ I distribution and kinematics within the disks of nearby galaxies, combining the baseline distributions of multiple existing (B, C, D) and hypothetical (E) JVLA configurations in one single array.

Previous surveys of nearby galaxies, such as THINGS [56] and HALOGAS [23], have concentrated on either obtaining a high spatial resolution or a high column density sensitivity — neither THINGS nor HALOGAS achieve both. Thanks to MeerKAT's combination of exquisite column density sensitivity, high spatial resolution and large field of view, MHONGOOSE will be the first survey that does not suffer from these limitations and that will therefore provide information on the processes driving the transformation and evolution of galaxies in the nearby universe at high resolution and to low column densities.

Specifically, it will be possible to investigate the low column-density H I, from the outskirts of the star-forming disks out into the far reaches of the dark matter halo. The observations, sensitive to column densities some two to three orders of magnitude lower than found in the main disk, will yield clues on gas flows in and out of the disk, accretion from the intergalactic medium (IGM), the fuelling of star formation, the connection with the cosmic web and even the possible existence of

\footnotetext{
${ }^{1}$ MeerKAT H I Observations of Nearby Galactic Objects; Observing Southern Emitters
} 
low-mass cold dark matter (CDM) halos. The higher resolution made possible by MeerKAT will resolve many of these phenomena, thus enabling a more detailed study of their internal structure, something not possible with previous surveys.

In the 2009/2010 proposal round, one of the projects submitted focused on the properties and evolution of magnetic fields in (amongst others) nearby galaxies (MeerQUITTENS; Bolton et al). While that project was not allocated survey time on MeerKAT, the team was encouraged to make use of commensal observations to incorporate MeerQUITTENS questions into the MeerKAT science programme. The scientific goals of MeerQUITTENS to determine the detailed 3D structure of gas and magnetic fields on sub-kpc scales as well as the relationship between magnetic fields and star formation is still highly relevant today, and MeerKAT promises uniquely powerful leverage on those questions. For these reasons we are incorporating a description of some of the relevant polarization science in this paper.

\section{Science Questions}

The science topics that will be addressed by MHONGOOSE are:

- the importance and effects of cold gas accretion;

- detection of the cosmic web;

- the relation between gas and star formation;

- the relation between dark and baryonic matter;

- the distribution of dark matter within galaxies;

- structure, strength and dynamical importance of magnetic fields.

A summary of the scientific background of some of these topics is given below.

\subsection{Accretion}

In the inner regions of spirals, time scales for consumption of gas by star formation are much smaller than a Hubble time, even though the star formation rate has been approximately constant over most of that time (e.g., [3]). Galactic disks can, in principle, be replenished by accreting gas-rich companion galaxies, but the slope of the H I mass function is not steep enough for small companions to supply larger galaxies with a substantial amount of gas for a sufficiently long time. This implies that spirals have to accrete directly from the IGM.

The presence of cold gas in the halos of our Milky Way and other galaxies has been known for some time (see, e.g., $[55,45,51,23])$. Some of this halo H I is likely to be part of a star formation driven "galactic fountain" [53]. This is suggested by the observation that some of the halo H I has a similar projected radial distribution to the star formation in the disk and that it has disk-like kinematics: rotating but lagging behind the main disk (see, e.g., [22]).

However, some of the $\mathrm{H}$ I complexes found outside the disks are counter-rotating with respect to the disk, so cannot have originated in it. Numerical simulations (e.g., [33]) predict that "fingers" of cooler gas from the IGM can penetrate the hot halos surrounding galaxies and deposit gas into 


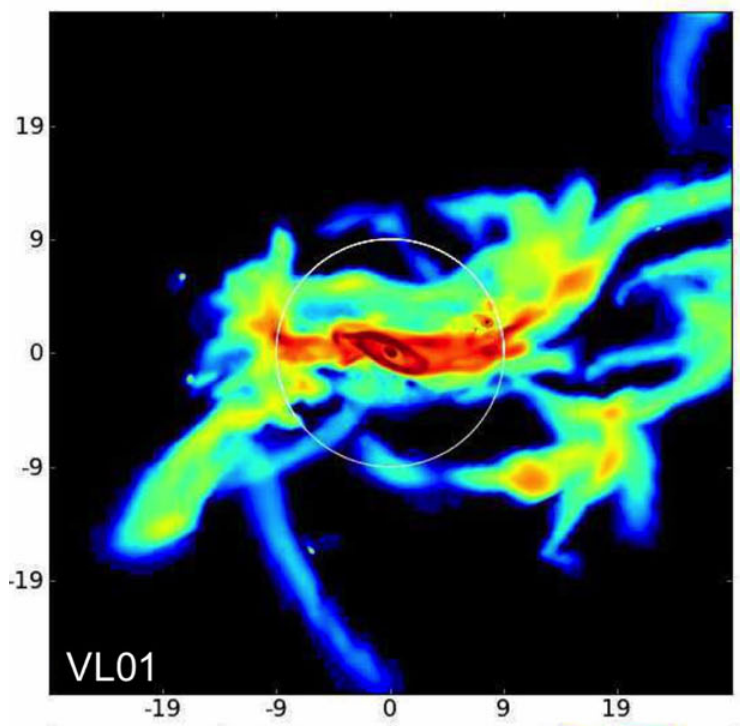

Figure 1: Visual impression of the morphology of simulated cold accretion features. The small dark brown ring in the center represents the main galaxy disk. The figure measures $\sim 60 \mathrm{kpc}$ on the side, the circle has a radius of $\sim 10 \mathrm{kpc}$. This area would fit within one MeerKAT primary beam for distances $D>4 \mathrm{Mpc}$. Figure taken from [13].

the disk. This process is called "cold accretion". Figure 1 gives a visual impression of typical cold accretion features around simulated galaxies. It is in the context of this cold accretion that the study of $\mathrm{H}$ I halos of galaxies is relevant: it could provide direct observations of the accretion of gas onto galaxies and forms a strong observational test for models of galaxy evolution.

The current state-of-the art survey of these H i halos is the WSRT HALOGAS project [23]. It has mapped 22 disk galaxies down to a column density limit of $\sim 10^{19} \mathrm{~cm}^{-2}$, i.e., an order of magnitude lower than typically found in the main $\mathrm{H}$ i disks. The first results of HALOGAS indicate that some galaxies have extended $\mathrm{H}$ I emission at these low levels (see Fig. 2), while others do not: extensive $\mathrm{H}$ I halos have been detected in about 12 of the 22 galaxies observed. It is possible that some of this gas is related to star formation and galactic fountain processes, but as discussed above, accretion cannot be excluded. The upper limit on the cold gas accretion rate as determined by HALOGAS is only $\sim 10 \%$ of the current star formation rate in the disk, suggesting most accretion must occur at lower neutral gas column densities (either because the column density is truly lower, or because a larger fraction of the gas is ionised).

The MHONGOOSE observations will probe a factor $\sim 50$ deeper in column density than HALOGAS and these deep observations will show how the low column density gas is connected with the cosmic web and where accretion occurs. Cold accretion is predicted to be the dominant process in galaxies with baryonic masses $\log \left(M_{\mathrm{bar}}\right)<10.3$ [33] corresponding to $\mathrm{H}$ I masses below a few times $10^{9} M_{\odot}$. The latter value is approximately equal to the average $\mathrm{H}$ I mass of the HALOGAS galaxies. To increase the chances of detecting the direct effects of cold accretion, the MHONGOOSE sample contains a larger number of galaxies with lower H I masses. 


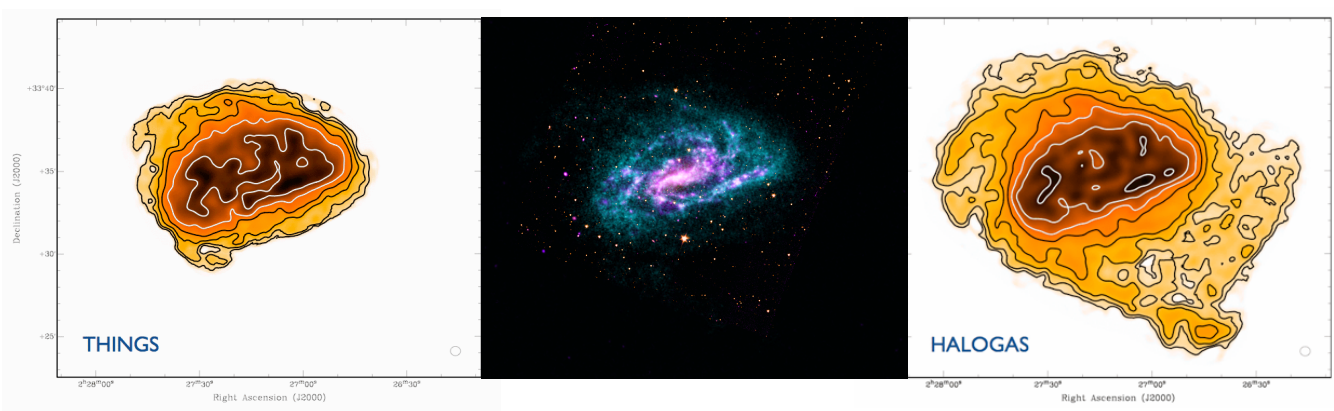

Figure 2: Comparison of shallow THINGS and deep HALOGAS observations of NGC 925 at the same spatial resolution. Left: The H I distribution of NGC 925 from THINGS, convolved to the WSRT resolution. Lowest contour at $9 \cdot 10^{19} \mathrm{~cm}^{-2}$, with each contour double the previous value. Center: False-color image of the baryonic components. Blue shows the H I distribution derived from THINGS, cyan the distribution of young stars derived from GALEX data. Right: The H I distribution as observed by the deep WSRT HALOGAS survey. Lowest contour $1.8 \cdot 10^{19} \mathrm{~cm}^{-2}$ with each contour double the previous value.

\subsection{Cosmic Web}

The cold accretion process described above delivers gas from the cosmic web into galaxies. This process is a prediction of high-resolution numerical models of structure formation (e.g., [14, 12]). These predict that most of the baryons at low redshift are in a warm-hot intergalactic medium (WHIM; T $=10^{5}-10^{7} \mathrm{~K}$ ), while $25 \%$ are in the $10^{4} \mathrm{~K}$ diffuse IGM, with only $25 \%$ condensed in galaxies and their gaseous halos. Due to the moderately high temperature in the IGM $\left(>10^{4} \mathrm{~K}\right)$, most of the gas in the cosmic web is ionised. To detect the cooler baryons in the cosmic web, a column density sensitivity of $\sim 10^{17-18} \mathrm{~cm}^{-2}$ is required [48]. Observationally the presence of cold gas around galaxies out to radii of at least $\sim 300 \mathrm{kpc}$ has been established (e.g., [5]).

MHONGOOSE will have enough sensitivity to reach these column density values. At a resolution of $90^{\prime \prime}$, the typical $3 \sigma$ column density sensitivity of the observations will be $\sim 5 \cdot 10^{17} \mathrm{~cm}^{-2}$. Stacking the H I profiles will push the effective column density sensitivity even lower by a factor of several. Pushing radio technology to the limit is the only way forward: optical telescopes will for the foreseeable future not be able to directly detect in emission the ionized gas which the $\mathrm{HI}$ traces.

These sensitivities are close to those obtained by very deep single-dish H I observations. The deepest of these are probably the observations by [6] (using the WSRT as a single dish) of the lowcolumn density features around and between M31 and M33. In these observations, the $3 \sigma$ limit over $16 \mathrm{~km} \mathrm{~s}^{-1}$ is $1.1 \cdot 10^{17} \mathrm{~cm}^{-2}$, but with an angular resolution of $\sim 49^{\prime}$.

A larger collection of very deep observations with the Green Bank Telescope (GBT) of the HALOGAS and THINGS galaxies has been obtained by D.J. Pisano (in prep.; see [47, 16]). These reach a $3 \sigma$ sensitivity of $\sim 6 \cdot 10^{17} \mathrm{~cm}^{-2}$. However, sheer column density sensitivity is not enough. For example, $[57,58]$ show that the diffuse low column density gas between M31 and M33 observed by [6] is resolved in several kpc-sized clouds when observed at higher spatial resolutions. The $90^{\prime \prime}$ MeerKAT beam measures a few kpc at the typical distance of our sample and is thus very well matched with the expected sizes of the cold accretion clouds. It is the powerful combination of column density sensitivity and spatial resolution that makes MeerKAT the ideal instrument for this work. 


\subsection{Gas and star formation}

MHONGOOSE will be able to make several key tests of interstellar medium (ISM) and star formation physics. Some of the work on this was also done by the THINGS survey [56] where obtaining a better understanding of the relation between gas and star formation was one of the main science goals [37]. The limited sensitivity of THINGS constrained these studies, however, to the optical disk only. The higher sensitivity of the MHONGOOSE observations, at a similar angular resolution, means these studies can now be extended to the outer parts of the disks. Molecular gas has been detected in the far outer parts of disks, so H I certainly changes phase there, leading to star formation [18].

In these outer parts, where $\mathrm{H} \mathrm{I}$ dominates, the ratio of UV to $\mathrm{H}$ I column density is the key tracer of the timescale and efficiency of star formation. We will compare this observable to proposed star formation timescales (e.g., [34, 37, 59]) and thresholds (e.g., [52, 15, 60]). With the MeerKAT observations providing the angular resolution to isolate specific conditions of the ISM, and the velocity resolution to separate warm and cold H I [29], MHONGOOSE promises to be a unique data set to study star formation in galaxy outskirts.

The MHONGOOSE sample includes edge-on galaxies, ensuring that deep and sensitive observations will be available for a detailed study of the vertical distribution of the $\mathrm{HI}$, associated flaring of the disk, the presence of gas above the disk as well as galactic fountain-type outflows due to star formation.

The large range in stellar disk mass in the MHONGOOSE sample will enable a study of the effect of increasing disk domination on the dark matter distributions in more massive galaxies. We will infer the distribution of dark matter and relate this to, e.g., disk mass density, scale length, disk spin/angular momentum, bulge/disk ratio, and star formation rate.

\section{Sample Definition and observing time}

In 2010, time was allocated to study a sample of 30 galaxies, chosen uniformly from bins in $\log \left(M_{\mathrm{HI}}\right)$ over the range $6<\log \left(M_{H I}\right)<11$, thus ensuring a flat distribution in $\log \left(M_{\mathrm{HI}}\right)$. The precursor sample from which our sample has been selected is based on SINGG (Survey for Ionization in Neutral Gas Galaxies; [42]). SINGG targeted $\sim 500$ HIPASS-detected nearby galaxies, also selected uniformly in bins of $\log \left(M_{H I}\right)$. The SINGG galaxies were selected to have a HIPASS peak flux $>50 \mathrm{mJy}$, a galactic latitude $|b|>30^{\circ}$, a projected distance from the center of the LMC $>10^{\circ}$ and a Galactic standard of rest velocity $>200 \mathrm{~km} \mathrm{~s}^{-1}$. $\mathrm{H} \alpha$, optical, infrared and ultraviolet data are available for the SINGG galaxies.

In selecting the MHONGOOSE galaxies, strongly interacting galaxies and dense group and cluster environments were avoided, since studies of isolated galaxies have shown that the gas captured from companion galaxies and galactic fountain processes (due to star formation and AGN) are minimized in these environments (AMIGA project: e.g., [19, 20, 38, 36, 50]). The contribution of the cold gas accretion should thus stand out more prominently this way. We further limited the sample to $\delta<-10^{\circ}$ and a distance $D<30 \mathrm{Mpc}$ (and excluded the MeerKAT Fornax survey region, PI Paolo Serra). The distance limit ensures that the beam of MeerKAT at the highest resolution is always smaller than $\sim 1 \mathrm{kpc}$ (comparable to the THINGS resolution). This left a target list of 

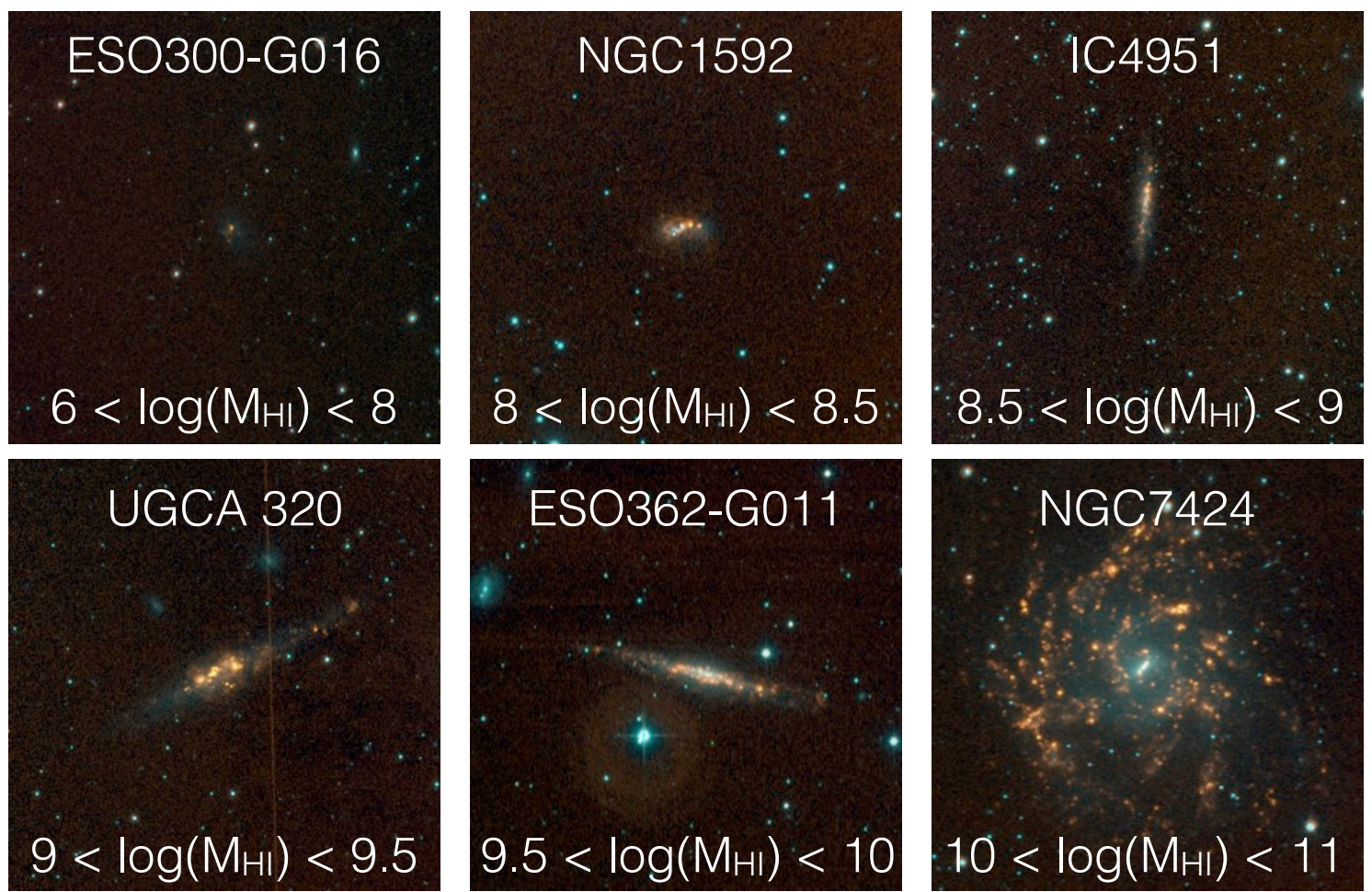

Figure 3: SINGG [42] images of one galaxy each from each of the $6 \mathrm{H}$ I mass bins. Orange/yellow shows the $\mathrm{H} \alpha$ emission, blue the optical $R$-band. A full set of images is available on the MHONGOOSE website at http: //mhongoose.astron.nl.

88 galaxies. These were divided in 6 bins of $\log \left(M_{\mathrm{HI}}\right)$, and in each bin 5 galaxies were selected, where each galaxy was selected to be either edge-on, face-on or with an intermediate inclination of 50-60 degrees. Face-on allows the best characterization of the morphology of the ISM, as well as determination of vertical motions. Edge-on allows an unambiguous characterization of the vertical structure of the ISM. The intermediate inclination range is optimal for determining rotation curves and kinematical modeling. Care was taken that a range in rotation velocity and star formation rate was covered ${ }^{2}$. A selection of SINGG images of the sample galaxies is shown in Fig. 3.

The desired column density limit for MHONGOOSE is $7.5 \cdot 10^{18} \mathrm{~cm}^{-2}$ at $3 \sigma$ over $16 \mathrm{~km} \mathrm{~s}^{-1}$ at $30^{\prime \prime}$ resolution. For the 2010 MeerKAT parameters, this corresponds to a noise of $0.074 \mathrm{mJy}$ beam ${ }^{-1}$ per $5 \mathrm{~km} \mathrm{~s}^{-1}$ channel assuming natural weighting ${ }^{3}$. With the current, updated MeerKAT parameters, this noise level is reached after $48^{h}$ on-source, assuming natural weighting. Assuming an overhead of $15 \%$ for set-up and calibration, results in a total time per galaxy of $55^{h}$. The total observing time to reach this sensitivity for the whole sample therefore becomes $30 \times 55^{h}=1650$ hours.

\footnotetext{
${ }^{2}$ For a more extensive description of the sample selection see the MHONGOOSE website at http://mhongoose. astron.nl.

${ }^{3}$ The calculation of the column density also includes a factor to take into account the increased noise due to tapering; cf. Fig. 4 and Table 3 in the original Call for Large Survey Projects [4].
} 


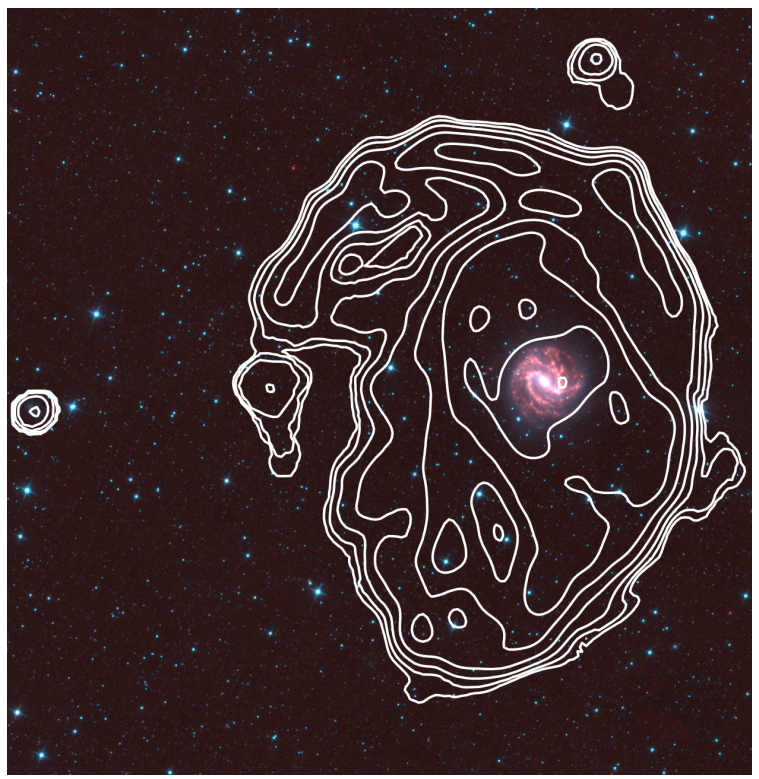

Figure 4: KAT-7 3-pointing mosaic of the extended H I distribution of M83 [25]. Contours show H I column densities and start at $5.6 \times 10^{18} \mathrm{~cm}^{-2}$ increasing by powers of 1.778 . The contours are overlaid on a threecolor WISE image. In this image, the blue channel is formed from W1 and W2, the green channel from W1, W2, and W3, and the red channel from W3 and W4. Each channel is displayed using a square-root stretch. The image measures 1.5 by 1.5 degrees.

\section{Comparison with previous surveys}

MHONGOOSE is designed to optimally make use of MeerKAT's unique capabilities: a high spatial and spectral resolution in combination with an excellent column density sensitivity and a wide field of view.

Most interferometric H I surveys of nearby galaxies in the last decade have concentrated on high angular resolution observations with a fairly modest column density sensitivity. These are surveys such as THINGS [56], LITTLE THINGS [28] and VLA-ANGST [46] which all reach column density limits of $\sim 10^{20} \mathrm{~cm}^{-2}$. The HALOGAS survey [23] is an exception to this. It used long integration times with the WSRT to reach column densities around $\sim 10^{19} \mathrm{~cm}^{-2}$, but at relatively low angular resolutions $\left(15^{\prime \prime}-30^{\prime \prime}\right)$.

To put MHONGOOSE in the context of these surveys, we compare their respective sensitivities in Fig. 5. Sensitivities of existing surveys have been taken from the source papers, and have all been converted to a $3 \sigma$ limit, integrated over a $16 \mathrm{~km} \mathrm{~s}^{-1}$ channel. It is clear that the observations of THINGS and its siblings only probe the high column density H I. Smoothing these data to lower resolutions increases their sensitivity somewhat, but the real jump in sensitivity is made by the HALOGAS survey. Recent, ultra-deep observations of the THINGS and HALOGAS galaxies obtained with the GBT $[47,16]$ are also indicated. These are some of the deepest H I observations ever done, but with a limited angular resolution $\left(\sim 9^{\prime}\right)$. IMAGINE (PI A. Popping) is a survey currently underway at the Australia Telescope Compact Array (ATCA) which also aims to image low-column density structures around nearby galaxies using the most compact configurations of ATCA. The angular resolution will be a few times better than the GBT observations. 


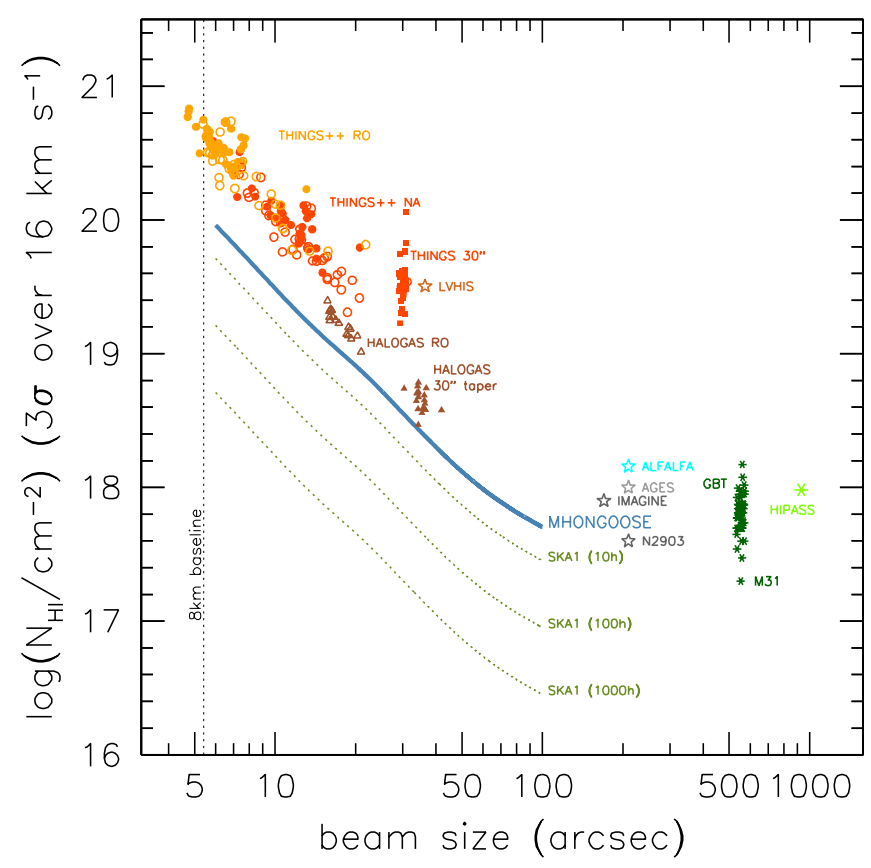

Figure 5: MHONGOOSE H I spectral line sensitivity versus other surveys. Light- and dark-orange filled circles show the sensitivities for individual galaxies in the THINGS survey, using robust $=0.5$ and natural weighting, respectively [56]. Light- and dark-orange open circles show the same for the LITTLE THINGS survey [28]. Dark-orange squares show the natural-weighted THINGS observations spatially smoothed to $30^{\prime \prime}$. Open and filled brown trianges show the individual HALOGAS [23] sensitivities at full resolution using robust $=0$, and tapered with a $30^{\prime \prime}$ taper, respectively. Light- and dark-gray stars indicate observations of NGC 2903 by [31], the AGES survey [43], and the IMAGINE survey. Dark-green stars indicate the GBT observations of THINGS and HALOGAS galaxies by Pisano (in prep.). The bottom green star indicates the deep M31 observation by [58] discussed in Sect. 4.2. Average sensitivities of the HIPASS, ALFALFA, and LVHIS surveys are also indicated. The MHONGOOSE sensitivity is indicated by the thick blue line. The dotted green lines show the expected sensitivities for SKA1-MID for observing times of $10^{h}, 100^{h}$ and $1000^{h}$.

Also shown is the expected sensitivity of the MHONGOOSE observations. It is abundantly clear that over the entire range of angular resolution shown here the MeerKAT observations will be superior ${ }^{4}$.

At the highest resolutions, MHONGOOSE will achieve the resolution of THINGS, but will be an order of magnitude deeper in column density. At resolutions of $\sim 1^{\prime}$ the observations reach the depth of the deepest GBT observations of nearby galaxies, but with an order of magnitude better angular resolution.

Using the known distances to the sample galaxies we can compare the column density sensitivities as a function of physical scale. For each galaxy in the MHONGOOSE sample we can calculate the column density as a function of physical resolution. As we vary the beam size from $8^{\prime \prime}$ to $90^{\prime \prime}$, each galaxy creates a track in the column density-physical resolution plane.

\footnotetext{
${ }^{4}$ The MHONGOOSE column densities take into account the increased noise due to tapering to the desired resolution, as derived using the most recent MeerKAT antenna configurations.
} 

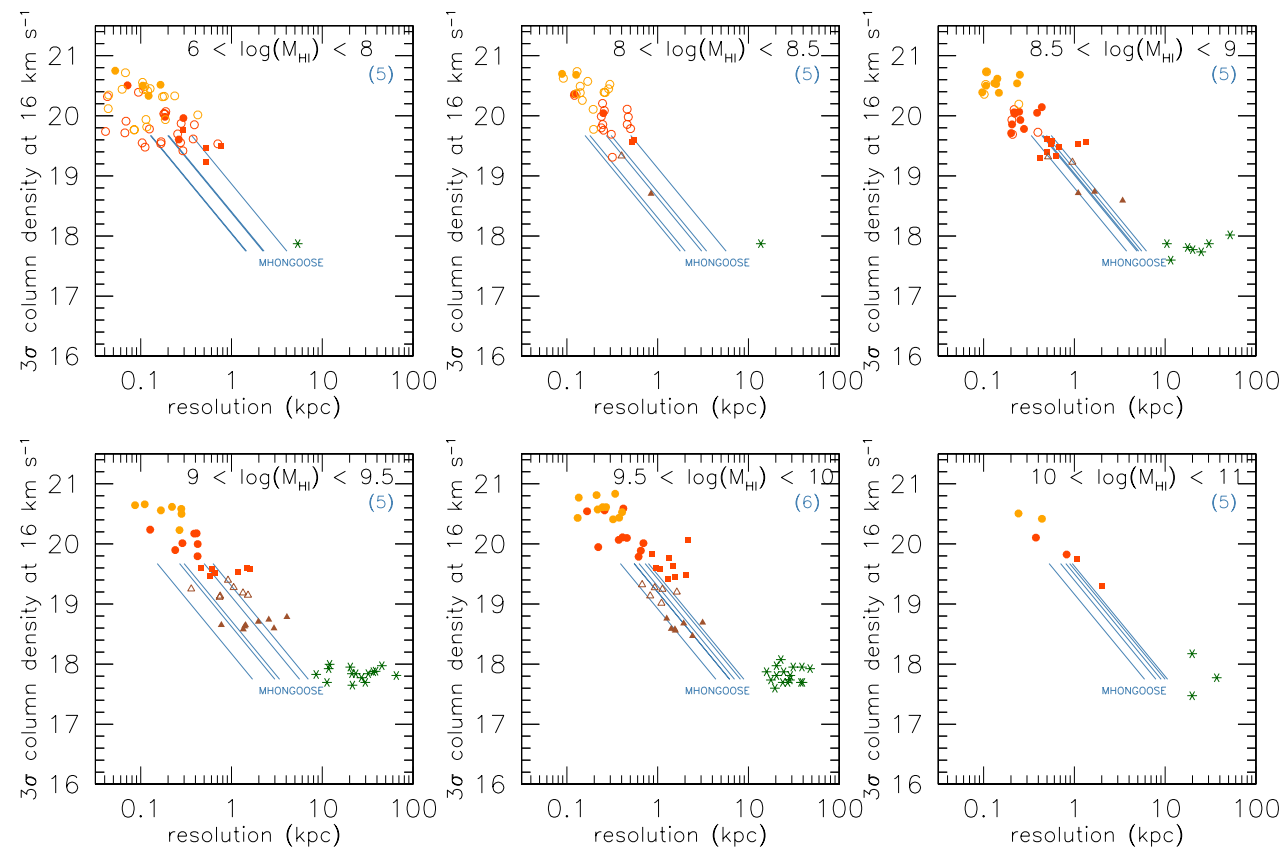

Figure 6: Column density sensitivity for individual MHONGOOSE galaxies as a function of linear resolution. Symbols are as in Fig. 5, and the blue lines show the sensitivity for each galaxy as the beam size is changed from $8^{\prime \prime}$ to $90^{\prime \prime}$. Panels show the galaxies in each H I mass bin. Note that in the lower centre panel 6 galaxies are shown, as one of the MHONGOOSE pointings covers two galaxies at different distances.

In Fig. 6 we show the tracks of the galaxies for each of the H I mass bins of the sample. We also show the galaxies from the other $\mathrm{H}$ I surveys with $\mathrm{H}$ I masses in that same bin. We see that the highest and lowest mass bins are very much unexplored at low column densities. The galaxies of HALOGAS mostly fall within the $9<\log \left(M_{H I}\right)<10$ mass bin, but even here the MHONGOOSE observations will probe an order of magnitude deeper for a given linear size.

\subsection{MHONGOOSE and low column densities}

Multiple independent lines of evidence show that the surface area subtended by $\mathrm{H}$ I at column densities near $10^{17} \mathrm{~cm}^{-2}$ is a factor of two larger than that seen at $10^{19} \mathrm{~cm}^{-2}[11,6,48,7]$. In other words, sizes of the $\mathrm{H}$ I disks will increase in area by a factor of two compared to the observations provided by surveys such as THINGS and HALOGAS.

The left panel in Fig. 7 shows the H I column density distribution function, or the likelihood that a line of sight encounters a certain column density. This shows that as one goes to lower column densities, the area covered by the $\mathrm{HI}$ increases, except around $\sim 10^{19} \mathrm{~cm}^{-2}$, where the slight dip in the function leads to the observed "edge" of the H I disk.

This is further illustrated in Fig. 7 (center), showing the fractional area $f_{A}=A\left(N_{H I}\right) / \mathrm{d} \log \left(N_{H I}\right)$ as a function of column density $N_{H I}$. We see that the disk hardly grows around $\sim 10^{19} \mathrm{~cm}^{-2}$, but then increases in size quickly again below a few times $10^{18} \mathrm{~cm}^{-2}$. HALOGAS probed the $10^{19}$ $\mathrm{cm}^{-2}$ regime and, while it picked up low column density gas in and around the disks of galaxies, it did not observe a significant increase in the size of the disk. MHONGOOSE will take us in a regime where disk size growth is very pronounced. The middle panel in Fig. 7 shows that the 

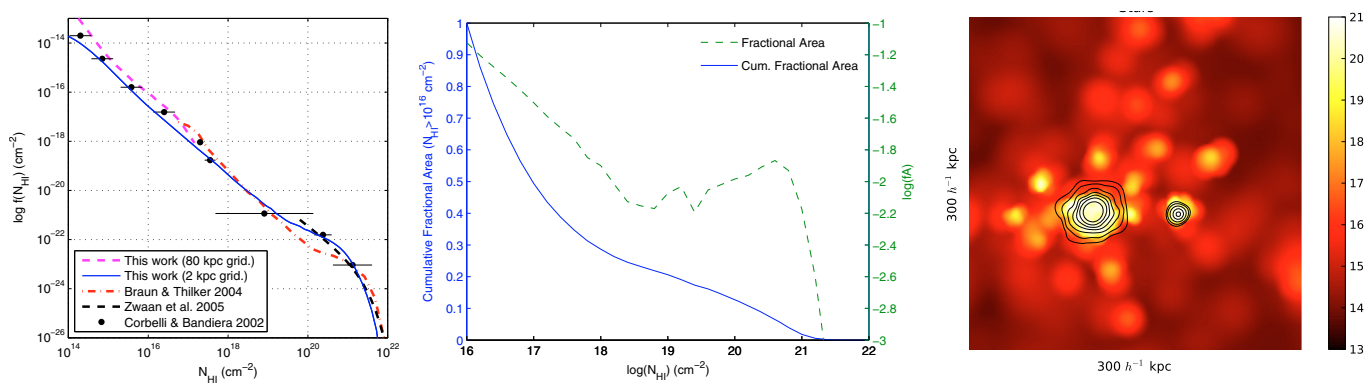

Figure 7: Figures from [48]. Left panel: the H I column density distribution function. Note the dip near $10^{19} \mathrm{~cm}^{-2}$ causing an "edge" to the H I disks of galaxies. Middle panel: fractional area (green) of a galaxy disk. The blue line shows the increase in area towards lower column densities. The area at $10^{16} \mathrm{~cm}^{-2}$ has been arbitrarily set to 1. Right panel: simulation of the morphology of the low column density material. The material is expected to be clumpy. The bar indicates the column density as $\log \left(N_{H I} /\left(\mathrm{cm}^{-2}\right)\right)$, contours indicate the locations of the stellar component.

area subtended by the $10^{17} \mathrm{~cm}^{-2}$ emission is twice as large as that of the $10^{19} \mathrm{~cm}^{-2}$ emission and $\sim 65 \%$ larger than that of the $10^{18} \mathrm{~cm}^{-2}$ emission.

However, detecting an increase in disk size is not the main goal of these observations. Rather, we want to characterize the morphology and dynamics of the ultra-low column density material as this is not expected to be in a smooth, homogeneously distributed disk. Simulations by [48] already indicate that the $10^{17} \mathrm{~cm}^{-2}$ material is likely distributed as clumps and clouds of a few kpc in size (see the right panel in Fig. 7). According to [48], these clouds are associated with accretion from the cosmic web. In a recent study, [58] have also shown that the material detected between M31 and M33 (originally detected as a smooth component by [6]) is clumpy at similar scales when observed at higher resolutions. This extremely deep observation is indicated separately in Fig. 5.

Very deep single pointings with the GBT by D.J. Pisano (priv. comm.), arranged in a sparsely sampled grid around NGC 2403, NGC 3198 and M31, and reaching column density limits of $\sim 10^{17} \mathrm{~cm}^{-2}$, confirm this. Aside from the limited resolution, sparsely sampled single pointings will, however, not be able to constrain the dynamics and morphology of the lowest column density gas. MeerKAT is currently the only telescope with the sensitivity and resolution that can address this and therefore uniquely placed to investigate this SKA1 key science question.

\subsection{Magnetic fields in the MHONGOOSE galaxies}

Magnetic fields are a crucial component of the ISM and the star formation cycle, but their detailed properties and role in galaxy evolution are still unclear. Observations of polarised synchrotron radiation in nearby galaxies (see, e.g., [1]) have given us a clear picture of the overall structure and energetics of galactic magnetic fields within the star forming disk. With modern radio techniques, we are now opening the window to the detailed 3D structure of gas and magnetic fields on scales relevant to constrain models of ISM physics (e.g., [40]). The relationship between detailed magnetic structure and star formation will be probed for the first time with the MHONGOOSE observations. We now appreciate that there exist deep degeneracies between source models with different combinations of synchrotron emission, magneto-ionic turbulence, and Faraday rotation (e.g., [27]). Through broadband synchrotron observations, these models can be distinguished (e.g., [24], Williams et al., in prep.). The key is in collecting polarimetric data over a wide 
range of $\lambda^{2}$ as even a modest increase can lead to strong leverage on distinct models of depolarisation effects. Techniques such as Rotation Measure Synthesis and QU-Fitting will be employed to derive maximum benefit from the MHONGOOSE polarimetry data.

For the nominal 0.9-1.6 GHz L-band continuum data, the instrumental precision in Faraday Rotation Measure (RM) is $23 \mathrm{rad} / \mathrm{m}^{2}$. This means that for well-detected polarised emission ( $\mathrm{S} / \mathrm{N}>$ 8) the effective RM precision is $\lesssim 1 \mathrm{rad} / \mathrm{m}^{2}$, sufficient to identify $\mathrm{kpc}$-scale fluctuations in magnetic field strength, within the star-forming ISM, of order $20 \mathrm{nG}$ (cf. the typical magnetic field strength of 1-10 $\mu \mathrm{G}$ ). MHONGOOSE will provide detailed measurements of magnetic field fluctuations in the ISM of the target galaxies.

MHONGOOSE will also provide a sensitive probe of the non-thermal component in the outer parts of galaxies (beyond the main star forming disk), tracing the large-scale morphology of magnetic fields and the magnetic connection to the IGM. This will be possible both through detection of diffuse polarised synchrotron radiation, as well as by identifying foreground contributions to the RM of background polarised radio galaxies. Thanks to the high sensitivity of MeerKAT's broadband continuum mode (typical sensitivity of $0.75 \mu \mathrm{Jy}_{\text {beam }}^{-1}$ for $48 \mathrm{~h}$ on-source, or 0.15 $\mu \mathrm{Jy} \mathrm{beam}^{-1}$ for the ultra-deep MHONGOOSE targets), diffuse synchrotron will be detected well outside the $25 \mathrm{mag} \operatorname{arcsec}^{-2}$ diameter $D_{25}$, and a typical background polarised source density of $\gtrsim 100 \mathrm{deg}^{-2}$ will be recovered at $\mathrm{S} / \mathrm{N} \geq 10$ (based on [49]). This kind of density is sufficient to work out the large-scale magnetic field properties even if no diffuse synchrotron is detected from the foreground (target) galaxy itself [54]. The MHONGOOSE observations will thus provide new constraints on the evolution of magnetic fields in galaxies, as well as the possible magnetisation of the IGM [1]. The MHONGOOSE sample is an excellent testbed for addressing questions regarding the evolution of magnetism, thanks to the broad diversity of galaxy properties that results from the adopted sample selection (e.g., rotational velocity and star formation rate, the two key ingredients of the galactic dynamo process; see. e.g., [2]).

\section{Summary}

MHONGOOSE is a MeerKAT Large Survey Project to map the neutral hydrogen distribution in a sample of 30 nearby galaxies. The sample covers all inclinations, H I masses from $\sim 10^{6}$ to $\sim 10^{11} M_{\odot}$, and luminosities from $M_{R} \sim 12$ to $M_{R} \sim 22$. It samples the complete range of conditions found in local galaxies: from prominent star forming disks all the way out to the little-explored low-column density gas far out in the dark matter halo. MHONGOOSE will be the first survey to provide a comprehensive inventory of the processes driving the transformation and evolution of galaxies in the nearby universe over 5 orders of magnitude in $\mathrm{H}$ I mass and column density. The MHONGOOSE data, in combination with data at other wavelengths, will provide the largest, most detailed and versatile legacy database of nearby galaxy observations that will not be surpassed until the SKA starts observing.

\section{References}

[1] Beck, R. 2015, A\&ARev, 24, 4

[2] Beck, R., Brandenburg, A., Moss, D., Shukurov, A., \& Sokoloff, D. 1996, ARA\&A, 34, 155 
[3] Bigiel, F., Leroy, A. K., Walter, F., et al. 2011, ApJL, 730, L13

[4] Booth, R. S., de Blok, W. J. G., Jonas, J. L., \& Fanaroff, B. 2009, arXiv:0910.2935

[5] Borisova, E., Cantalupo, S., Lilly, S. J., et al. 2016, arXiv:1605.01422

[6] Braun, R., \& Thilker, D. A. 2004, A\&A, 417, 421

[7] Braun, R. 2012, ApJ, 749, 87

[8] Braun, R., Bourke, T., Green, J., Wagg, J. 2014, SKA document SKA-TEL-SKO-0000122

[9] Carignan, C., Frank, B. S., Hess, K. M., et al. 2013, AJ, 146, 48

[10] Carignan, C. 2016, Cosmic Web research with KAT-7, MeerKAT \& FAST, Frontiers in Radio Astronomy and FAST Early Sciences Symposium 2015 (FRA2015), 29-31 July 2015, Guiyang, China, ASP Conf. Ser., 502, 55

[11] Corbelli, E., \& Bandiera, R. 2002, ApJ, 567, 712

[12] Crain, R. A., Bahe, Y. M., Lagos, C. d. P., et al. 2016, arXiv:1604.06803

[13] Danovich, M., Dekel, A., Hahn, O., Ceverino, D., \& Primack, J. 2015, MNRAS, 449, 2087

[14] Davé, R., Hernquist, L., Katz, N., \& Weinberg, D. H. 1999, ApJ, 511, 521

[15] de Blok, W. J. G., \& Walter, F. 2006, AJ, 131, 363

[16] de Blok, W. J. G., Keating, K. M., Pisano, D. J., et al. 2014, A\&A, 569, A68

[17] de Blok, E., Fraternali, F., Heald, G., et al. 2015, Advancing Astrophysics with the Square Kilometre Array (AASKA14), 129

[18] Dessauges-Zavadsky, M., Verdugo, C., Combes, F., \& Pfenniger, D. 2014, A\&A, 566, A147

[19] Espada, D., Muñoz-Mateos, J. C., Gil de Paz, A., et al. 2011, ApJj, 736, 20

[20] Espada, D., Verdes-Montenegro, L., Huchtmeier, W. K., et al. 2011, A\&A, 532, A117

[21] Frank, B. S., de Blok, W. J. G., Walter, F., Leroy, A., \& Carignan, C. 2016, AJ, 151, 94

[22] Fraternali, F., Oosterloo, T., Sancisi, R., \& van Moorsel, G. 2001, ApJL, 562, L47

[23] Heald, G., Józsa, G., Serra, P., et al. 2011, A\&A, 526, A118

[24] Heald, G., Beck, R., de Blok, W. J. G., et al. 2015, Advancing Astrophysics with the Square Kilometre Array (AASKA14), 106

[25] Heald, G., de Blok, W. J. G., Lucero, D., et al. 2016, MNRAS, 462, 1238

[26] Hess, K. M., Jarrett, T. H., Carignan, C., Passmoor, S. S., \& Goedhart, S. 2015, MNRAS, 452,1617

[27] Horellou, C., \& Fletcher, A. 2014, MNRAS, 441, 2049

[28] Hunter, D. A., Ficut-Vicas, D., Ashley, T., et al. 2012, AJ, 144, 134

[29] Ianjamasimanana, R., de Blok, W. J. G., Walter, F., \& Heald, G. H. 2012, AJ, 144, 96

[30] Ianjamasimanana, R., de Blok, W. J. G., Walter, F., et al. 2015, AJ, 150, 47

[31] Irwin, J. A., Hoffman, G. L., Spekkens, K., et al. 2009, ApJ, 692, 1447

[32] Józsa, G. I. G., Kenn, F., Klein, U., \& Oosterloo, T. A. 2007, A\&A, 468, 731

[33] Kereš, D., Katz, N., Weinberg, D. H., \& Davé, R. 2005, MNRAS, 363, 2 
[34] Krumholz, M. R., \& Tan, J. C. 2007, ApJ, 654, 304

[35] Krumholz, M. R., McKee, C. F., \& Tumlinson, J. 2009, ApJ, 693, 216

[36] Leon, S., Verdes-Montenegro, L., Sabater, J., et al. 2008, A\&A, 485, 475

[37] Leroy, A. K., Walter, F., Brinks, E., Bigiel, F., de Blok, W. J. G., Madore, B., \& Thornley, M. D. 2008, AJ, 136, 2782

[38] Lisenfeld, U., Verdes-Montenegro, L., Sulentic, J., et al. 2007, A\&A, 462, 507

[39] Lucero, D. M., Carignan, C., Elson, E. C., et al. 2015, MNRAS, 450, 3935

[40] Mao, S. A., McClure-Griffiths, N. M., Gaensler, B. M., et al. 2015, Highlights of Astronomy, 16, 403

[41] McKee, C. F., \& Krumholz, M. R. 2010, ApJ, 709, 308

[42] Meurer, G. R., et al. 2006, ApJS, 165, 307

[43] Minchin, R. F., Momjian, E., Auld, R., et al. 2010, AJ, 140, 1093

[44] Mogotsi, K. M., de Blok, W. J. G., Caldú-Primo, A., et al. 2016, AJ, 151, 15

[45] Oosterloo, T., Fraternali, F., \& Sancisi, R. 2007, AJ, 134, 1019

[46] Ott, J., Stilp, A. M., Warren, S. R., et al. 2012, AJ, 144, 123

[47] Pisano, D. J. 2014, AJ, 147, 48

[48] Popping, A., Davé, R., Braun, R., \& Oppenheimer, B. D. 2009, A\&A, 504, 15

[49] Rudnick, L., \& Owen, F. N. 2014, ApJ, 785, 45

[50] Sabater, J., Verdes-Montenegro, L., Leon, S., Best, P., \& Sulentic, J. 2012, A\&A, 545, A15

[51] Sancisi, R., Fraternali, F., Oosterloo, T., \& van der Hulst, T. 2008, A\&ARev, 15, 189

[52] Schaye, J. 2004, ApJ, 609, 667

[53] Shapiro, P. R., \& Field, G. B. 1976, ApJ, 205, 762

[54] Stepanov, R., Arshakian, T. G., Beck, R., Frick, P., \& Krause, M. 2008, A\&A, 480, 45

[55] Wakker, B. P., \& van Woerden, H. 1997, ARA\&A, 35, 217

[56] Walter, F., Brinks, E., de Blok, W. J. G., et al. 2008, AJ, 136, 2563-2647

[57] Wolfe, S. A., Pisano, D. J., Lockman, F. J., McGaugh, S. S., \& Shaya, E. J. 2013, Nature, 497, 224

[58] Wolfe, S. A., Lockman, F. J., \& Pisano, D. J. 2016, ApJ, 816, 81

[59] Wong, T. 2009, ApJ, 705, 650

[60] Yang, C.-C., Gruendl, R. A., Chu, Y.-H., Mac Low, M.-M., \& Fukui, Y. 2007, ApJ, 671, 374 